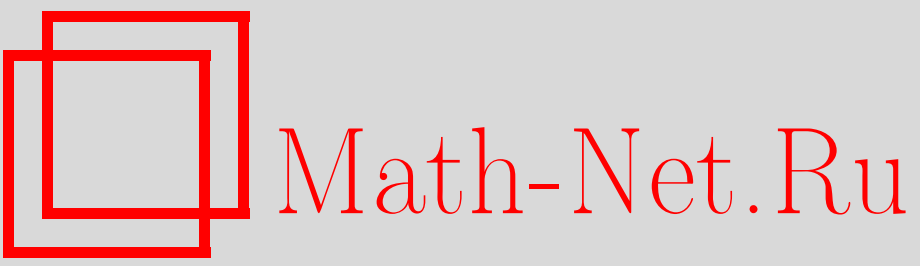

Х. Брезис, Как распознать постоянные функции. Связь с пространствами Соболева, УМН, 2002, том 57, выпуск 4, 59-74

DOI: https://doi.org/10.4213/rm533

Использование Общероссийского математического портала Math-Net.Ru подразумевает, что вы прочитали и согласны с пользовательским соглашением

http://www.mathnet.ru/rus/agreement

Параметры загрузки:

IP: 34.239 .49 .27

26 апреля 2023 г., 13:59:12 


\section{КАК РАСПОЗНАТЬ ПОСТОЯННЫЕ ФУНКЦИИ. СВЯЗЬ С ПРОСТРАНСТВАМИ СОБОЛЕВА}

Х. БРЕЗИС

Дается критерий принадлежности функции $f \in L^{p}$ пространству $W^{1, p}(p>1)$ и пространству $B V(p=1)$. Обсуждаются различные интегральные условия, при выполнении которых измеримая функция является константой.

Библиографоия: 17 названий.

\section{СОДЕРЖАНИЕ}

$\S 1$. Введение

$\S 2$. Новая характеризация соболевских пространств ................. 63

$\S 3$. Возвращение к постоянным функциям …......................... 71

$\S 4$. Другой подход. Связь с VMO ................................... 72

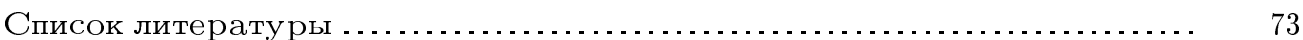

\section{$\S$ 1. Введение}

Большинство идей в этой статье происходят из цикла совместных работ автора с Ж. Бургэном, Я. Ли, П. Миронеску и Л. Ниренбергом (см. [4]-[7], [10], [11]). Тем не менее мы представим немного другое изложение, в частности, упрошая некоторые доказательства.

Мы начнем со следующего предложения.

ПРЕДЛОЖЕНИЕ 1. Пусть $\Omega$ - связное открытое множество в $\mathbb{R}^{N}$, и пусть $f: \Omega \rightarrow \mathbb{R}-$ измеримая функиия такая, что

$$
\int_{\Omega} \int_{\Omega} \frac{|f(x)-f(y)|}{|x-y|^{N+1}} d x d y<\infty
$$

Тогда $f$ является константой.

Исходной мотивацией для подобного утверждения были следуюшие два направления. 
(i) Единственность поднятия. Для данной (измеримой) функции $u: \Omega \rightarrow \mathbb{C}$ такой, что $|u|=1$ почти всюду, сушествует много поднятий $\varphi$, т.е. $u=e^{i \varphi}$. Если $\varphi_{1}, \varphi_{2}-$ два поднятия, то

$$
k(x)=\frac{1}{2 \pi}\left(\varphi_{1}(x)-\varphi_{2}(x)\right): \Omega \rightarrow \mathbb{Z} .
$$

При дополнительных предположениях иногда удается доказать, что $k$ является noстоянной функшией. Например, если $\varphi_{1}, \varphi_{2}-$ непрерывны и $\Omega$ - связна, то $k-$ постоянная функция. Мы хотим сказать, что на самом деле условие непрерьвности может быть ослаблено до некоторого условия другого типа, такого как (1), которое намного более естественно при работе с соболевскими пространствами (см. замечание 3 ).

(ii) Теория степени для классов разрывных отображений. Возможность определения степени для отображений, принадлежаших пространствам Соболева (см. [9], [11]), основьвается на факте, что $\operatorname{deg} h_{t}(\cdot)$ не изменяется при гомотопии $h_{t}(\cdot)$, когда $t$ пробегает $[0,1]$ (или, в общем случае, связное параметрическое пространство $\Lambda$ ). Подобные заключения могут быть возможны в ситуациях, когда зависимость от $t$ не обязана быть непрерьвной.

ЗАмЕчАнИЕ 1. Предложение 1 легко формулируется, однако мы не знаем прямого, элементарного доказательства. Наше доказательство не очень сложно, но требует использования теории соболевских пространств.

ЗАмЕчАнИЕ 2. Условие связности является, очевидно, сушественньм. Утверждение предложения 1 остается справедливым, если $N+1$ в неравенстве (1) заменить на любое $q \geqslant N+1$. В самом деле, достаточно доказать предложение 1 в случае, когда область $\Omega$ является шаром $B$ (обший случай следует в силу связности); тогда

$$
\frac{1}{|x-y|^{N+1}} \leqslant \frac{C}{|x-y|^{q}} \quad \forall x, y \in B .
$$

(У тверждение, тем не менее, остается верным в некоторых несвязных областях, например в $\Omega=G \backslash \Sigma$, где $G$ - связно, а $\Sigma$ - замкнутое множество нулевой меры. Возникает интересная задача - изучить, в каких несвязных областях справедливо утверждение предложения 1.)

С другой стороны, если $N+1$ в (1) заменить на $q<N+1$, то утверждение перестанет быть верньм. Действительно, для любой липшицевой функции на $B$ вьполнено:

$$
\int_{B} \int_{B} \frac{|f(x)-f(y)|}{|x-y|^{q}} d x d y \leqslant C \int_{B} \int_{B} \frac{d x d y}{|x-y|^{q-1}}<\infty,
$$

поскольку $q<N+1$.

Из предложения 1 можно вывести множество разнообразных следствий. Мы приведем несколько из них.

СЛЕДСТВИЕ 1 . Пусть $\Omega$ - связное открытое множество в $\mathbb{R}^{N}$ и $f: \Omega \rightarrow \mathbb{Z}$ измеримая функция такая, что

$$
\int_{\Omega} \int_{\Omega} \frac{|f(x)-f(y)|^{p}}{|x-y|^{N+1}} d x d y<\infty
$$

при некотором $1 \leqslant p<\infty$. Тогда $f$ является постоянной функцией. 
ДокАЗАТЕЛЬСтво. Достаточно заметить, что

$$
|f(x)-f(y)|^{p} \geqslant|f(x)-f(y)|,
$$

поскольку $f(x)-f(y) \in \mathbb{Z}$.

ЗАмечАниЕ 3 . При $p>1$ условие (2) говорит, что $f$ лежит в пространстве Соболева (с дробными производными) $W^{s, p}$ с $s=1 / p$ (см., например, [1]). Поэтому мы можем утверждать, что при условии $s p \geqslant 1$ любая функция, лежашая в $W^{s, p}(\Omega ; \mathbb{Z})$, является константой. Заметим, что условие $s p \geqslant 1$ значительно слабее, чем условие $s p>N$, которое влечет (по теореме вложения Соболева), что $f$-непрерьвна. Следствие 1 при $p=2$ и $s=1 / 2$ можно найти в [15; лемма 1.1] (авторы этой работы приписывают его Винеру в случае $N=2$ ). В [3] получено подобное утверждение при более сильном предположении $s p>1$.

СледСтвиЕ 2. Предположим, что $\Omega$ - связное открытое множество в $\mathbb{R}^{N}$ и $A$ - произвольное измеримое подмножество такое, что

$$
\int_{A} \int_{c_{A}} \frac{d x d y}{|x-y|^{N+1}}<\infty
$$

Тогда либо множество $A$, либо множество $(\Omega \backslash A)$ имеет меру нуль.

Для доказательства достаточно применить предложение 1 с $f=\chi_{A}$ - характеристической функцией множества $A$. Заметим, что показатель $N+1$ в (3) опять оптимален. Действительно, пусть $A$ - произвольное подмножество $\Omega$ с гладкой границей, тогда неравенство (3) вьполняется, если $N+1$ заменить на любое $q<N+1$ (достаточно рассмотреть случай, когда граница $\partial A$ - плоская, и сделать прямое вычисление).

Сформулируем ряд обобшений предложения 1.

ПреДЛОЖЕнИЕ 2 . Пусть $\Omega$ - связное открытое множество в $\mathbb{R}^{N}$ u $f: \Omega \rightarrow \mathbb{R}$ - измеримая функиия такая, ито

$$
\int_{\Omega} \int_{\Omega} \frac{|f(x)-f(y)|^{p}}{|x-y|^{N+p}} d x d y<\infty
$$

для некоторого $1 \leqslant p<\infty$. Тогда $f-$ константа.

(Предложение 1 соответствует случаю $p=1$.)

Следующим обобщением является

ПрЕДЛОЖЕНИЕ 3 . Пусть $\Omega$ - связное открытое множество в $\mathbb{R}^{N}$ и $f: \Omega \rightarrow \mathbb{R}$ - измеримая функция такая, что

$$
\int_{\Omega} \int_{\Omega} \frac{|f(x)-f(y)|^{p}}{|x-y|^{p}} \psi(|x-y|) d x d y<\infty,
$$

где $p \geqslant 1$ и функиия $\psi \in L_{\mathrm{loc}}^{1}(0, \infty), \psi \geqslant 0$, удовлетворяет условию

$$
\int_{0}^{1} \psi(r) r^{N-1} d r=\infty
$$

Тогда $f$ - константа.

(Предложение 2 соответствует случаю $\psi(r)=r^{-N}$.)

Приведем одно важное обобщение предложения 2. 
ПРЕДЛОЖЕНИЕ 4. Пусть $\Omega$ - связное открытое множество в $\mathbb{R}^{N}$ и $f: \Omega \rightarrow \mathbb{R}$ - измеримая функиия такая, что

$$
\int_{\Omega} \int_{\Omega} \frac{|f(x)-f(y)|^{p}}{|x-y|^{N+p-\varepsilon}} d x d y=o\left(\frac{1}{\varepsilon}\right) \quad \text { npu } \quad \varepsilon \rightarrow 0
$$

m.e.

$$
\lim _{\varepsilon \rightarrow 0} \varepsilon \int_{\Omega} \int_{\Omega} \frac{|f(x)-f(y)|^{p}}{|x-y|^{N+p-\varepsilon}} d x d y=0
$$

для некоторого $p \geqslant 1$. Тогда $f-$ константа.

ЗАмЕчАниЕ 4 . В случае, когда $\Omega$ ограничено, условие (7), очевидно, намного слабее, чем условие (4), которое означает, что

$$
\int_{\Omega} \int_{\Omega} \frac{|f(x)-f(y)|^{p}}{|x-y|^{N+p-\varepsilon}} d x d y=O(1) \text { при } \varepsilon \rightarrow 0 .
$$

С другой стороны, условие (7) оптимально, так как для любой липшицевой функщии $f$ на $\Omega$

$$
\int_{\Omega} \int_{\Omega} \frac{|f(x)-f(y)|^{p}}{|x-y|^{N+p-\varepsilon}} d x d y=O\left(\frac{1}{\varepsilon}\right)
$$

поскольку

$$
\int_{0}^{1} \frac{1}{r^{N-\varepsilon}} r^{N-1} d r=\frac{1}{\varepsilon} .
$$

Сформулируем итоговое обобщение, которое вплотную подводит нас к связи с соболевскими пространствами.

ТеОрема 1. Пусть $\Omega$ - связное открытое множество в $\mathbb{R}^{N}$ и $f: \Omega \rightarrow \mathbb{R}$ измеримая функиия. Пусть $\left(\rho_{\varepsilon}\right)_{\varepsilon>0}$ - последовательность функиий такая, что

$$
\begin{gathered}
\rho_{\varepsilon} \in L_{\mathrm{loc}}^{1}(0, \infty), \quad \rho_{\varepsilon} \geqslant 0, \\
\int_{0}^{\infty} \rho_{\varepsilon}(r) r^{N-1} d r=1 \quad \forall \varepsilon>0, \\
\text { для кажддого } \quad \delta>0 \quad \lim _{\varepsilon \rightarrow 0} \int_{\delta}^{\infty} \rho_{\varepsilon}(r) r^{N-1} d r=0 .
\end{gathered}
$$

Предположим, что для некоторого $p \geqslant 1$

$$
\lim _{\varepsilon \rightarrow 0} \int_{\Omega} \int_{\Omega} \frac{|f(x)-f(y)|^{p}}{|x-y|^{p}} \rho_{\varepsilon}(|x-y|) d x d y=0
$$

Тогда $f$ - константа.

Заметим, что предложение 4 является следствием теоремы 1, если выбрать

$$
\rho_{\varepsilon}(r)= \begin{cases}\varepsilon r^{-N+\varepsilon}, & r<1, \\ 0, & r>1 .\end{cases}
$$


Предложение 3 также является следствием теоремы 1 при выборе

$$
\rho_{\varepsilon}(r)= \begin{cases}0 & \text { при } r<\varepsilon, \\ a_{\varepsilon} \psi(r) & \text { при } \varepsilon<r<1, \\ 0 & \text { при } r>1,\end{cases}
$$

где

$$
a_{\varepsilon}=\left(\int_{\varepsilon}^{1} \psi(r) r^{N-1} d r\right)^{-1} \rightarrow 0 \text { при } \varepsilon \rightarrow 0 .
$$

Заметим, что ввиду (5) и (13)

$$
\int_{\Omega} \int_{\Omega} \frac{|f(x)-f(y)|^{p}}{|x-y|^{p}} \rho_{\varepsilon}(|x-y|) d x d y \leqslant C a_{\varepsilon} \rightarrow 0 \text { при } \varepsilon \rightarrow 0 .
$$

Чтобы доказать теорему 1 , мы должны сделать небольшое отступление в теорию соболевских пространств.

\section{§. Новая характеризация соболевских пространств}

Для простоты мы начнем со случая всего $\mathbb{R}^{N}$. Пусть $f \in L^{p}\left(\mathbb{R}^{N}\right), 1<p<\infty$. Хорошо известно (см., например, [8; предложение IX.3]), что если $f \in W^{1, p}\left(\mathbb{R}^{N}\right)$, то

$$
\int_{\mathbb{R}^{N}}|f(x+h)-f(x)|^{p} d x \leqslant|h|^{p} \int_{\mathbb{R}^{N}}|\nabla f|^{p} d x \text { для каждого } h \in \mathbb{R}^{N} .
$$

Обратно, если $f \in L^{p}\left(\mathbb{R}^{N}\right)$ и сушествует такая константа $C$, что

$$
\int_{\mathbb{R}^{N}}|f(x+h)-f(x)|^{p} d x \leqslant C|h|^{p} \text { при } h \rightarrow 0,
$$

то $f \in W^{1, p}\left(\mathbb{R}^{N}\right)$.

При $p=1$ надо заменить $W^{1,1}$ на $B V$ - пространство функций из $L^{1}$, производные которых (в смысле теории распределений) - ограниченные меры Радона. Таким образом, $f \in B V$ тогда и только тогда, когда

$$
\int_{\mathbb{R}^{N}}|f(x+h)-f(x)| d x \leqslant C|h| \text { при }|h| \rightarrow 0,
$$

и в этом случае неравенство (16) справедливо при всех $h \in \mathbb{R}^{N}$ с константой $C=$ $\int|\nabla f| d x$. В частности, если $\rho_{\varepsilon}$ удовлетворяет $(9),(10)$ и $f \in W^{1, p}$, мы получаем

$$
\int_{\mathbb{R}^{N}} \rho_{\varepsilon}(|h|) d h \int_{\mathbb{R}^{N}} \frac{|f(x+h)-f(x)|^{p}}{|h|^{p}} d x \leqslant C \text { при } \varepsilon \rightarrow 0,
$$

TaK KaK

$$
\int_{\mathbb{R}^{N}} \rho_{\varepsilon}(|h|) d h=\sigma_{N} \int_{0}^{\infty} \rho_{\varepsilon}(r) r^{N-1} d r=\sigma_{N},
$$

где $\sigma_{N}=\left|S^{N-1}\right|$. 
Сделав замену переменных в (17), получаем

$$
\int_{\mathbb{R}^{N}} \int_{\mathbb{R}^{N}} \frac{|f(x)-f(y)|^{p}}{|x-y|^{p}} \rho_{\varepsilon}(|x-y|) d x d y \leqslant C \text { при } \varepsilon \rightarrow 0 .
$$

Аналогично, если $f \in B V$, то

$$
\int_{\mathbb{R}^{N}} \int_{\mathbb{R}^{N}} \frac{|f(x)-f(y)|}{|x-y|} \rho_{\varepsilon}(|x-y|) d x d y \leqslant C \text { при } \varepsilon \rightarrow 0 .
$$

Важньм моментом является то, что условие (18) (соответственно (19)), на самом деле, характеризует принадлежность пространству $W^{1, p}$ при $p>1$ (соответственно $B V)$.

Теорема 2. Предположим, ито $f \in L^{p}\left(\mathbb{R}^{N}\right), p>1$, и удовлетворяет (18). Пусть семейство функиий $\left(\rho_{\varepsilon}\right)$ удовлетворяет условиям (9)-(11). Тогда $f \in W^{1, p}$ $u$

$$
\lim _{\varepsilon \rightarrow 0} \int_{\mathbb{R}^{N}} \int_{\mathbb{R}^{N}} \frac{|f(x)-f(y)|^{p}}{|x-y|^{p}} \rho_{\varepsilon}(|x-y|) d x d y=K_{p, N} \int_{\mathbb{R}^{N}}|\nabla f|^{p} d x
$$

где $K_{p, N}$ зависит лишь от $р$ и $N$.

Аналогично, при $p=1$ имеем следуюшую теорему.

ТеОрема 3. Предположим, ито $f \in L^{1}\left(\mathbb{R}^{N}\right)$ удовлетворяет (19). Пусть семейство функиий $\left(\rho_{\varepsilon}\right)$ удовлетворяет условиям (9)-(11). Тогда $f \in B V u$

$$
\lim _{\varepsilon \rightarrow 0} \int_{\mathbb{R}^{N}} \int_{\mathbb{R}^{N}} \frac{|f(x)-f(y)|}{|x-y|} \rho_{\varepsilon}(|x-y|) d x d y=K_{1, N} \int_{\mathbb{R}^{N}}|\nabla f| d x,
$$

где интеграл в правой части обозначает полную вариацию меры $\nabla f$.

У теоремы 3 имеется интересное

СлЕДСТВИЕ 3. Пусть $A$ - ограниченное измеримое множество в $\mathbb{R}^{N}$. Тогда А имеет ограниченный периметр (в смысле де Джсиорджи) тогда и только тогда, когда

$$
\int_{A} \int_{c_{A}} \frac{1}{|x-y|} \rho_{\varepsilon}(|x-y|) d x d y \leqslant C \quad \text { npu } \quad \varepsilon \rightarrow 0
$$

и в этом случае

$$
\lim _{\varepsilon \rightarrow 0} \int_{A} \int_{c_{A}} \frac{1}{|x-y|} \rho_{\varepsilon}(|x-y|) d x d y=K_{1, N} \operatorname{Per}(A)
$$


ДокАЗАТЕЛЬСТво тЕОРемЫ 2. Первоначальное доказательство теоремы 2 может быть найдено в [6]. Здесь мы изложим более простое, сообщенное нам И. Стейном. Предположим, что функция $f \in L^{p}$ удовлетворяет (18). Рассмотрим последовательность гладких приближений

$$
f_{\delta}=\gamma_{\delta} \star f,
$$

где $\left(\gamma_{\delta}\right)$ - гладкая аппроксимативная единица. Заметим, что (18) останется справедливым, если функцию $f$ заменить на любой ее сдвиг $\left(\tau_{h} f\right)(x)=f(x+h)$. Кроме того, неравенство (18) выдерживает операцию взятия выпуклой оболочки. Следовательно, $f_{\delta}$ удовлетворяет (18) с той же константой $C$. Таким образом, справедливо неравенство

$$
\int_{\mathbb{R}^{N}} \int_{\mathbb{R}^{N}} \frac{\left|f_{\delta}(x)-f_{\delta}(y)\right|^{p}}{|x-y|^{p}} \rho_{\varepsilon}(|x-y|) d x d y \leqslant C,
$$

где $C$ - не зависит от $\varepsilon$ и $\delta$.

Далее воспользуемся следуюшим утверждением. Пусть функция $g \in C^{2}\left(\mathbb{R}^{N}\right)$ удовлетворяет условию

$$
\int_{\mathbb{R}^{N}} \int_{\mathbb{R}^{N}} \frac{|g(x)-g(y)|^{p}}{|x-y|^{p}} \rho_{\varepsilon}(|x-y|) d x d y \leqslant C \text { при } \varepsilon \rightarrow 0,
$$

где последовательность $\rho_{\varepsilon}$ удовлетворяет (9)-(11). Тогда

$$
\int_{\mathbb{R}^{N}}|\nabla g(x)|^{p} d x \leqslant \frac{C}{K_{p, N}},
$$

где $C$ берется из $(24)$ и

$$
K_{p, N}=\int_{S^{N-1}}|(\sigma \cdot e)|^{p} d \sigma, \quad e \in S^{N-1} .
$$

Доказательство неравенства (25). Возьмем произвольный компакт $K$ в $\mathbb{R}^{N}$. Для всех $x \in K$ и $|h| \leqslant 1$ имеем

$$
|g(x+h)-g(x)-h \cdot \nabla g(x)| \leqslant C_{K}|h|^{2} .
$$

Из неравенства (24) имеем

$$
\int_{K} d x \int_{|h| \leqslant 1} \frac{|g(x+h)-g(x)|^{p}}{|h|^{p}} \rho_{\varepsilon}(|h|) d h \leqslant C .
$$

Из (27) получаем

$$
|h \cdot \nabla g(x)| \leqslant|g(x+h)-g(x)|+C_{K}|h|^{2},
$$

и, следовательно, для каждого $\theta>0$

$$
|h \cdot \nabla g(x)|^{p} \leqslant(1+\theta)|g(x+h)-g(x)|^{p}+C_{\theta, K}|h|^{2 p} .
$$


Используя (28), получаем:

$$
\int_{K} d x \int_{|h| \leqslant 1} \frac{|(h \cdot \nabla g(x))|^{p}}{|h|^{p}} \rho_{\varepsilon}(|h|) d h \leqslant(1+\theta) C+C_{\theta, K}|K| \int_{|h| \leqslant 1}|h|^{p} \rho_{\varepsilon}(|h|) d h .
$$

Заметим, что для каждого вектора $V \in \mathbb{R}^{N}$ справедливо равенство

$$
\int_{|h| \leqslant 1} \frac{|(h \cdot V)|^{p}}{|h|^{p}} \rho_{\varepsilon}(|h|) d h=K_{p, N}|V|^{p} \int_{0}^{1} \rho_{\varepsilon}(r) r^{N-1} d r .
$$

Также заметим, что условия (10) и (11) влекут

$$
\lim _{\varepsilon \rightarrow 0} \int_{|h| \leqslant 1}|h|^{p} \rho_{\varepsilon}(|h|) d h=0 .
$$

Переходя в неравенстве $(29)$ к пределу при $\varepsilon \rightarrow 0$, мы получаем

$$
K_{p, N} \int_{K}|\nabla g(x)|^{p} d x \leqslant(1+\theta) C .
$$

Поскольку (30) выполняется для каждого $\theta>0$ и для каждого компакта $K$ (с независящей от $\theta$ и $K$ константой $C$ ), мы получаем (25) и, как следствие,

$$
K_{p, N} \int_{\mathbb{R}^{N}}|\nabla g(x)|^{p} d x \leqslant \liminf _{\varepsilon \rightarrow 0} \int_{\mathbb{R}^{N}} \int_{\mathbb{R}^{N}} \frac{|g(x)-g(y)|^{p}}{|x-y|^{p}} \rho_{\varepsilon}(|x-y|) d x d y .
$$

$\mathrm{C}$ другой стороны, если $g \in C_{0}^{2}\left(\mathbb{R}^{N}\right)$, то

$$
|g(x+h)-g(x)| \leqslant|h \cdot \nabla g(x)|+C^{\prime}|h|^{2} \quad \forall x \in \mathbb{R}^{N}, \quad \forall h \in \mathbb{R}^{N} .
$$

Следовательно,

$$
|g(x+h)-g(x)|^{p} \leqslant(1+\theta)|h \cdot \nabla g(x)|^{p}+C_{\theta}^{\prime}|h|^{2 p} .
$$

Умножив на $\rho_{\varepsilon}(|h|) /|h|^{p}$ и проинтегрировав по множеству $\left\{(x, h) \in \mathbb{R}^{2 N}: x\right.$ или $x+h \in$ $\operatorname{supp} g\}$, получаем:

$$
\begin{aligned}
& \int_{\mathbb{R}^{N}} d x \int_{\mathbb{R}^{N}} \frac{|g(x+h)-g(x)|^{p}}{|h|^{p}} \rho_{\varepsilon}(|h|) d h \\
& \quad \leqslant(1+\theta) \int_{\mathbb{R}^{N}} K_{p, N}|\nabla g(x)|^{p} d x+2 C_{\theta}^{\prime}|\operatorname{supp} g| \int_{\mathbb{R}^{N}}|h|^{p} \rho_{\varepsilon}(|h|) d h .
\end{aligned}
$$

Переходя к пределу сначала при $\varepsilon \rightarrow 0$, а затем при $\theta \rightarrow 0$, получаем

$$
\limsup _{\varepsilon \rightarrow 0} \int_{\mathbb{R}^{N}} d x \int_{\mathbb{R}^{N}} \frac{|g(x+h)-g(x)|^{p}}{|h|^{p}} \rho_{\varepsilon}(|h|) d h \leqslant \int_{\mathbb{R}^{N}} K_{p, N}|\nabla g(x)|^{p} d x .
$$

Из (31) и (32) следует, что для любой функции $g \in C_{0}^{2}\left(\mathbb{R}^{N}\right)$

$$
\lim _{\varepsilon \rightarrow 0} \int_{\mathbb{R}^{N}} \int_{\mathbb{R}^{N}} \frac{|g(x)-g(y)|^{p}}{|x-y|^{p}} \rho_{\varepsilon}(|x-y|) d x d y=K_{p, N} \int_{\mathbb{R}^{N}}|\nabla g|^{p} d x .
$$

Поскольку $C_{0}^{2}\left(\mathbb{R}^{N}\right)$ плотно в $W^{1, p}\left(\mathbb{R}^{N}\right)$, то отсюда нетрудно получить (используя (14)), что (20) вьполняется для каждой функции $f \in W^{1, p}\left(\mathbb{R}^{N}\right)$.

Теперь мы можем завершить доказательство теоремы 2 . Предполагая, что $f \in$ $L^{p}\left(\mathbb{R}^{N}\right)$ удовлетворяет (18), и применяя неравенство $(25)$ к $g=f_{\delta}$, мы получаем, что

$$
\int_{\mathbb{R}^{N}}\left|\nabla f_{\delta}\right|^{p} d x \leqslant \frac{C}{K_{p, N}},
$$

где $C$ - константа неравенства (18). Переходя к пределу в неравенстве (33) при $\delta \rightarrow 0$, мы получаем, что $f \in W^{1, p}$. 
ДОКАЗАТЕЛЬСТВО ТЕОРЕМЫ 3 . Пусть $f \in L^{1}\left(\mathbb{R}^{N}\right)$ удовлетворяет условию (19). Повторяя доказательство предыдущей теоремы, мы получаем, что

$$
\int_{\mathbb{R}^{N}}\left|\nabla f_{\delta}\right| d x \leqslant \frac{C}{K_{1, N}} .
$$

Поэтому $f \in B V$ и

$$
\int_{\mathbb{R}^{N}}|\nabla f| d x \leqslant \frac{C}{K_{1, N}} .
$$

Иными словами, мы доказали, что

$$
K_{1, N} \int_{\mathbb{R}^{N}}|\nabla f| d x \leqslant \liminf _{\varepsilon \rightarrow 0} \int_{\mathbb{R}^{N}} \int_{\mathbb{R}^{N}} \frac{|f(x)-f(y)|}{|x-y|} \rho_{\varepsilon}(|x-y|) d x d y .
$$

С другой стороны, используя (16), легко заключить, что для каждой функции $f \in B V$ вьполнено

$$
\int_{\mathbb{R}^{N}} \int_{\mathbb{R}^{N}} \frac{|f(x)-f(y)|}{|x-y|} \rho_{\varepsilon}(|x-y|) d x d y \leqslant \widetilde{K}_{N} \int_{\mathbb{R}^{N}}|\nabla f| d x .
$$

$\mathrm{K}$ сожалению, константа $\widetilde{K}_{N}$ в $(35)$ не совпадает с $K_{1, N}$. Соотношение (21), очевидно, вьполняется для функций $f \in C_{0}^{2}\left(\mathbb{R}^{N}\right)$. Однако из этого мы не можем сразу заключить, что оно вьполняется для каждой функции $f \in B V$, поскольку пространство $C_{0}^{2}\left(\mathbb{R}^{N}\right)$ не плотно в $B V$.

Остается доказать, что для каждой функции $f \in B V\left(\mathbb{R}^{N}\right)$

$$
\limsup _{\varepsilon \rightarrow 0} \int_{\mathbb{R}^{N}} \int_{\mathbb{R}^{N}} \frac{|f(x)-f(y)|}{|x-y|} \rho_{\varepsilon}(|x-y|) d x d y \leqslant K_{1, N} \int_{\mathbb{R}^{N}}|\nabla f| d x .
$$

Это неравенство доказано в [12] с использованием некоторых новых идей, которые мы не будем здесь воспроизводить.

ЗАмечАниЕ 5. Утверждения, подобные теоремам 2 и 3 , справедливы если $\mathbb{R}^{N}$ заменить на гладкую ограниченную область $\Omega$ в $\mathbb{R}^{N}$. Однако они не верны для произвольных ограниченных областей $\Omega$ в случае, если $\partial \Omega-$ не гладкая. Тем не менее, для произвольных областей $\Omega$ справедливо неравенство

$$
K_{p, N} \int_{\Omega}|\nabla f|^{p} d x \leqslant \liminf _{\varepsilon \rightarrow 0} \int_{\Omega} \int_{\Omega} \frac{|f(x)-f(y)|^{p}}{|x-y|^{p}} \rho_{\varepsilon}(|x-y|) d x d y .
$$

Однако при $p>1$ может случиться так, что $f \in W^{1, p}(\Omega)$ (т.е. левая часть в (36) конечна), тогда как правая часть в (36) - бесконечна. Приведем пример. Пусть $\Omega=$ $D \backslash \Sigma$, где $D$ - диск $\left(\right.$ в $\left.\mathbb{R}^{2}\right)$ и $\Sigma$ - разрез. Пусть $f$ - гладкая функция в $\Omega$, которая разрывна на разрезе (например, две разные константы на разных сторонах разреза). Ясно, что $f \in W^{1, p}(\Omega)$, но правая часть в $(36)$ - бесконечна. Это следует из того, что

$$
\int_{\Omega} \int_{\Omega} \cdots=\int_{D} \int_{D} \cdots
$$

и если бы правая часть в (36) была конечна, то мы могли бы заключить, что $f \in$ $W^{1, p}(D)$ (по теореме 2 ), что, очевидно, не так. Этот пример наводит на следуюший открытьй вопрос. 
ОтКрЫТАЯ ЗАДАчА 1 . Пусть $\Omega \subset \mathbb{R}^{N}$ - ограниченное связное множество (не обязательно гладкое). Пусть $\delta(x, y)$ обозначает геодезическое расстояние в $\Omega$. Пусть $f \in L^{p}(\Omega)$ удовлетворяет условию

$$
\int_{\Omega} \int_{\Omega} \frac{|f(x)-f(y)|^{p}}{\delta(x, y)^{p}} \rho_{\varepsilon}(\delta(x, y)) d x d y \leqslant C \text { при } \varepsilon \rightarrow 0 .
$$

Следует ли из этого, что $f \in W^{1, p}$, и если да, то верно ли, что

$$
\lim _{\varepsilon \rightarrow 0} \int_{\Omega} \int_{\Omega} \frac{|f(x)-f(y)|^{p}}{\delta(x, y)^{p}} \rho_{\varepsilon}(\delta(x, y)) d x d y=K_{p, N} \int_{\Omega}|\nabla f|^{p} d x ?
$$

ЗАмечАниЕ 6 . Характеризация пространства $W^{1, p}$ (соответственно $B V$ ), данная теоремой 2 (соответственно 3), мотивирует следующее определение соболевских пространств для отображений $f: M \rightarrow \widetilde{M}$ метрических пространств, где $M-$ снабжено мерой $\mu$ :

$$
\iint \frac{\widetilde{d}(f(x), f(y))^{p}}{d(x, y)^{p}} \rho_{\varepsilon}(d(x, y)) d \mu(x) d \mu(y) \leqslant C \text { при } \varepsilon \rightarrow 0 .
$$

Заметим, что в условиях (10) и (11) участвует размерность $N$, но вместо нее можно рассмотреть $\lim _{r \rightarrow 0}\left|\log \mu\left(B_{r}(x)\right)\right| /|\log r|$. Было бы интересно изучить свойства таких отображений (теоремы вложения Соболева и т. п.) и сравнить это определение с другими (см. [16], [14], [2], а также библиографию в этих работах).

ЗАмечАниЕ 7 . Справедливы аналоги теорем 2 и 3 , когда $\Omega-$ гладкая ограниченная область в $\mathbb{R}^{N}$. Например, справедлива

Теорема 2'. Пусть $f \in L^{p}(\Omega)$ удовлетворяет

$$
\int_{\Omega} \int_{\Omega} \frac{|f(x)-f(y)|^{p}}{|x-y|^{p}} \rho_{\varepsilon}(|x-y|) d x d y \leqslant C \quad \text { npu } \quad \varepsilon \rightarrow 0,
$$

где для последовательности $\rho_{\varepsilon}$ выполнены условия (9)-(11). Тогда $f \in W^{1, p}(\Omega)$ $u$

$$
\lim _{\varepsilon \rightarrow 0} \int_{\Omega} \int_{\Omega} \frac{|f(x)-f(y)|^{p}}{|x-y|^{p}} \rho_{\varepsilon}(|x-y|) d x d y=K_{p, N} \int_{\Omega}|\nabla f|^{p} d x .
$$

СХема ДОКАЗАТЕльСтвА. Допустим, что выполнено (37). Используя стандартную технику отражения относительно гранищы и домножения на срезающую функцию, можно построить функцию $\widetilde{f}$ на $\mathbb{R}^{N}$ с компактным носителем такую, что $\tilde{f}=f$ на $\Omega$ и

$$
\int_{\mathbb{R}^{N}} \int_{\mathbb{R}^{N}} \frac{|\widetilde{f}(x)-\tilde{f}(y)|^{p}}{|x-y|^{p}} \rho_{\varepsilon}(|x-y|) d x d y \leqslant C^{\prime} \quad \text { при } \varepsilon \rightarrow 0 .
$$

По теореме 2 мы получаем, что $\tilde{f} \in W^{1, p}\left(\mathbb{R}^{N}\right)$ и, следовательно, $f \in W^{1, p}(\Omega)$.

Далее мы показываем, что для $f \in C^{2}(\bar{\Omega})$ справедливо неравенство

$$
\int_{\Omega} \int_{\Omega} \frac{|f(x)-f(y)|^{p}}{|x-y|^{p}} \rho_{\varepsilon}(|x-y|) d x d y \leqslant C(\Omega) \int_{\Omega}|\nabla f|^{p} d x .
$$

После этого неравенство (38) следует для $f \in C^{2}(\bar{\Omega})$. Заключение теоремы $2^{\prime}$ получается из соображений плотности. 
ЗАмЕчАниЕ 8. Некоторые конкретные выборы последовательности $\rho_{\varepsilon}$ представляют особьй интерес. Приведем несколько из них.

А) Выбор 1:

$$
\rho_{\varepsilon}(r)= \begin{cases}\frac{\varepsilon}{r^{N-\varepsilon}}, & 0<r<1 \\ 0, & r>1\end{cases}
$$

Такой выбор влечет

СЛЕДСТВИЕ 4. Пусть $\Omega$ - гладкая открытая область в $\mathbb{R}^{N}$. Пусть функиия $f \in L^{p}(\Omega)$ удовлетворяет неравенству

$$
\varepsilon \int_{\Omega} \int_{\Omega} \frac{|f(x)-f(y)|^{p}}{|x-y|^{N+p-\varepsilon}} d x d y \leqslant C \quad \text { npu } \quad \varepsilon \rightarrow 0 .
$$

Тогда $f \in W^{1, p}(\Omega) u$

$$
\lim _{\varepsilon \rightarrow 0} \varepsilon \int_{\Omega} \int_{\Omega} \frac{|f(x)-f(y)|^{p}}{|x-y|^{N+p-\varepsilon}} d x d y=K_{p, N} \int_{\Omega}|\nabla f|^{p} d x .
$$

Напомним, что стандартные соболевские пространства с дробными производными $W^{s, p}, 0<s<1,1<p<\infty$, снабжаются (полу) нормой Гальярдо

$$
\|f\|_{W^{s, p}}^{p}=\int_{\Omega} \int_{\Omega} \frac{|f(x)-f(y)|^{p}}{|x-y|^{N+s p}} d x d y .
$$

Хорошо известно, что величина $\|f\|_{W^{s, p}}$ не сходится к $\|f\|_{W^{1, p}}$ при $s \uparrow 1$; на самом деле, по предложению 2 она стремится к $\infty$ (если только $f$ не константа). Теперь же ввиду следствия 4 мы можем утверждать, что

$$
\lim _{s \uparrow 1}(1-s)\|f\|_{W^{s, p}}^{p}=\frac{K_{p, N}}{p} \int_{\Omega}|\nabla f|^{p} d x .
$$

Это “восстанавливает" $W^{1, p}$ в качестве непрерьвного предела пространств $W^{s, p}$ при $s \uparrow 1$ при условии, что мы используем нормы $(1-s)^{1 / p}\|f\|_{W^{s, p}}$ в пространствах $W^{s, p}$.

В) Выбор 2:

$$
\rho_{\varepsilon}(r)= \begin{cases}\frac{N}{\varepsilon^{N}}, & \text { если } r<\varepsilon \\ 0, & \text { если } r>\varepsilon .\end{cases}
$$

Этот выбор влечет

$$
\lim _{\varepsilon \rightarrow 0} \frac{1}{\varepsilon^{N}} \int_{\substack{\Omega \\|x-y|<\varepsilon}} \frac{|f(x)-f(y)|^{p}}{|x-y|^{p}} d x d y=\frac{K_{p, N}}{N} \int_{\Omega}|\nabla f|^{p} d x .
$$

Модификация этого выбора:

$$
\rho_{\varepsilon}(r)= \begin{cases}\frac{(N+p) r^{p}}{\varepsilon^{N+p}}, & r<\varepsilon, \\ 0, & r>\varepsilon,\end{cases}
$$


влечет

$$
\lim _{\varepsilon \rightarrow 0} \frac{1}{\varepsilon^{N+p}} \int_{\substack{\Omega \\|x-y|<\varepsilon}} \int_{\Omega}|f(x)-f(y)|^{p} d x d y=\frac{K_{p, N}}{N+p} \int_{\Omega}|\nabla f|^{p} d x .
$$

Аналогично можно получить

$$
\lim _{\varepsilon \rightarrow 0} \frac{1}{\varepsilon^{N+p}} \int_{\substack{\Omega \\ \varepsilon<|x-y|<2 \varepsilon}} \int_{\Omega}|f(x)-f(y)|^{p} d x d y=\widetilde{K}_{p, N} \int_{\Omega}|\nabla f|^{p} d x .
$$

С) Выбор 3:

$$
\rho_{\varepsilon}(r)= \begin{cases}0, & r<\varepsilon, \\ \frac{1}{|\log \varepsilon| r^{N}}, & \varepsilon<r<1, \\ 0, & r>1\end{cases}
$$

Этот выбор влечет

$$
\lim _{\varepsilon \rightarrow 0} \frac{1}{|\log \varepsilon|} \int_{\Omega} \int_{\Omega} \frac{|f(x)-f(y)|^{p}}{|x-y|^{N+p}} d x d y=K_{p, N} \int_{\Omega}|\nabla f|^{p} d x .
$$

D) Выбор 4: Пусть $\gamma \in L_{\text {loc }}^{1}(0,+\infty)$ - такая неотрицательная функция, что

$$
\int_{0}^{\infty} \gamma(r) r^{N+p-1} d r=1
$$

Выбирая

$$
\rho_{\varepsilon}(r)=\frac{1}{\varepsilon^{N+p}} \gamma\left(\frac{r}{\varepsilon}\right) r^{p}
$$

получаем

$$
\lim _{\varepsilon \rightarrow 0} \frac{1}{\varepsilon^{N+p}} \int_{\Omega} \int_{\Omega}|f(x)-f(y)|^{p} \gamma\left(\frac{|x-y|}{\varepsilon}\right) d x d y=K_{p, N} \int_{\Omega}|\nabla f|^{p} d x
$$

для каждой $f \in W^{1, p}$ (при $p>1$ ) и для каждой $f \in B V$ (при $p=1$ ). Применяя это равенство при $p=1$ (случай $B V)$ к $f=\chi_{A}$, мы получим новую характеризачию множеств ограниченного периметра: измеримое множество $A \subset \Omega$ имеет ограниченный периметр тогда и только тогда, когда

$$
\frac{1}{\varepsilon^{N+1}} \int_{A} \int_{c_{A}} \gamma\left(\frac{|x-y|}{\varepsilon}\right) d x d y \leqslant C \text { при } \varepsilon \rightarrow 0
$$

и в этом случае

$$
\lim _{\varepsilon \rightarrow 0} \frac{1}{\varepsilon^{N+1}} \int_{A} \int_{c_{A}} \gamma\left(\frac{|x-y|}{\varepsilon}\right) d x d y=K_{1, N} \operatorname{Per}(A) .
$$




\section{§3. Возвращение к постоянным функциям}

Все результаты $\S 1$ - непосредственные следствия утверждений $\S 2$, примененные к шару $B \subset \Omega$. Получаем, что $f$ - постоянна в любом шаре $B$ и, следовательно, $f-$ постоянна на $\Omega$, поскольку $\Omega$ - связна.

Заметим, что условие

$$
\lim _{\varepsilon \rightarrow 0} \int_{B} \int_{B} \frac{|f(x)-f(y)|}{|x-y|} \rho_{\varepsilon}(|x-y|) d x d y=0
$$

влечет, во-первых, что $f \in B V$, и, во-вторых, что $\nabla f=0$. Таким образом, $f$ - постоянная функция.

В случае же, когда $p>1$ и $f$ принимает значения в $\mathbb{Z}$, достаточно предположить, что

$$
\int_{B} \int_{B} \frac{|f(x)-f(y)|^{p}}{|x-y|^{p}} \rho_{\varepsilon}(|x-y|) d x d y \leqslant C \text { при } \varepsilon \rightarrow 0 .
$$

В самом деле, (50) влечет, что $f \in W^{1, p}$ (обратим внимание, что при $p=1$ (50) влечет лишь, что $f \in B V)$. Теперь можно воспользоваться тем, что функция $f$ принимает значения в $\mathbb{Z}$, и заключить, что $f$ - постоянная функция. Действительно, запишем

$$
\Omega=\bigcup_{k \in \mathbb{Z}} A_{k}
$$

где $A_{k}=\{x \in \Omega: f(x)=k\}$, и воспользуемся хорошо известньм результатом Стампаккья (см., например, лемму 7.7 в [13]), утверждающим, что $\nabla f=0$ п.в. в $A_{k}$. Следовательно, $\nabla f=0$ почти всюду на $\Omega$.

Приведем альтернативное доказательство. Из (50) и условия $f: \Omega \rightarrow \mathbb{Z}$ следует, что

$$
\int_{\Omega} \int_{\Omega} \frac{|f(x)-f(y)|}{|x-y|} \frac{\rho_{\varepsilon}(|x-y|)}{|x-y|^{p-1}} d x d y \leqslant C .
$$

Из чего легко следует, что

$$
\lim _{\varepsilon \rightarrow 0} \int_{\Omega} \int_{\Omega} \frac{|f(x)-f(y)|}{|x-y|} \rho_{\varepsilon}(|x-y|) d x d y=0 .
$$

Таким образом, $f$ - постоянная функция.

Имеются интересные обобшения некоторых приведенных результатов на случай, когда отношение $\frac{|f(x)-f(y)|^{p}}{|x-y|^{p}}$ заменено более обшим выражением $\omega\left(\frac{|f(x)-f(y)|}{|x-y|}\right)$. Мы приведем два таких обобшения, сообшенные нам Р. Игнатом, В. Ли и А. Понсе.

Теорема 4. Пусть $\omega:[0, \infty) \rightarrow[0, \infty)$ - непрерывная функиия такая, что $\omega(0)=0, \omega(t)>0 \quad \forall t u$

$$
\int_{1}^{\infty} \frac{\omega(t)}{t^{2}} d t=\infty
$$

Предположим, что функиия $f \in L^{1}(\Omega)$ удовлетворяет

$$
\int_{\Omega} \int_{\Omega} \omega\left(\frac{|f(x)-f(y)|}{|x-y|}\right) \frac{d x d y}{|x-y|^{N}}<\infty .
$$

Тогда $f$ - постоянная функиия. 
Теорема 5. Пусть $\omega:[0, \infty) \rightarrow[0, \infty)$ - непрерывная функиия такая, что $\omega(0)=0 u$

$$
\lim _{t \rightarrow \infty} \frac{\omega(t)}{t}=\alpha>0
$$

Предположим, что функиия $f \in L^{1}(\Omega)$ удовлетворяет

$$
\int_{\Omega} \int_{\Omega} \omega\left(\frac{|f(x)-f(y)|}{|x-y|}\right) \rho_{\varepsilon}(|x-y|) d x d y \leqslant C \quad \text { npu } \quad \varepsilon \rightarrow 0 .
$$

Tогда $f \in B V u$

$\lim _{\varepsilon \rightarrow 0} \int_{\Omega} \int_{\Omega} \omega\left(\frac{|f(x)-f(y)|}{|x-y|}\right) \rho_{\varepsilon}(|x-y|) d x d y=\int_{\Omega} \bar{\omega}\left(\left|\nabla f_{a c}\right|\right) d x+\alpha K_{1, N} \int_{\Omega}\left|\nabla f_{s}\right| d x$,

əде $\bar{\omega}(t)=\int_{S^{N-1}} \omega(t|\sigma \cdot e|) d \sigma u \nabla f=\nabla f_{a c}+\nabla f_{s}-$ разложение Радона-Никодима мерьл $\nabla f$.

Следующий вопрос пока остается открытым:

ОТКРЫТАя ЗАДАчА 2 . Пусть $\Omega$ - (гладкая) связная, ограниченная область в $\mathbb{R}^{N}$. Пусть $f: \Omega \rightarrow \mathbb{R}$ - непрерьвная (или даже гёльдерова) функция. Пусть $\omega:[0, \infty) \rightarrow$ $[0, \infty)$ - непрерьвная функция такая, что $\omega(0)=0$ и $\omega(t)>0$ при $t>0$. (Здесь (51) может не выполняться.) Предположим, что

$$
\int_{\Omega} \int_{\Omega} \omega\left(\frac{|f(x)-f(y)|}{|x-y|}\right) \frac{|d x d y|}{|x-y|^{N}}<\infty
$$

Можно ли из этого заключить, что $f$ - постоянная функция?

\section{§4. Другой подход. Связь с VMO}

Сначала мы напомним определение пространства $\operatorname{VMO}(\Omega ; \mathbb{R})$ (функций с исчезающей средней осцилляцией). Мы говорим, что функция $f \in \operatorname{VMO}(\Omega ; \mathbb{R})$, если $f \in$ $L_{\text {loc }}^{1}(\Omega ; \mathbb{R})$ и

$$
\lim _{\varepsilon \rightarrow 0} \frac{1}{\left|B_{\varepsilon}(x)\right|^{2}} \int_{B_{\varepsilon}(x)} \int_{B_{\varepsilon}(x)}|f(y)-f(z)| d y d z=0 \text { равномерно по } x \in \Omega .
$$

Пусть $\Omega$ - связное (открытое) множество в $\mathbb{R}^{N}$, и пусть $f \in \operatorname{VMO}(\Omega ; \mathbb{Z})$. Тогда $f-$ постоянная функция. Это было уже отмечено в $[10 ; \S$ I.5, часть 2$]$. В самом деле, если мы положим

$$
\bar{f}_{\varepsilon}(x)=\frac{1}{\left|B_{\varepsilon}(x)\right|} \int_{B_{\varepsilon}(x)} f(y) d y
$$

тогда $\operatorname{dist}\left(\bar{f}_{\varepsilon}(x), \mathbb{Z}\right) \rightarrow 0$ равномерно в $\Omega($ см. [10; $\left.§ .1]\right)$ и, таким образом, найдется целое число $k_{\varepsilon}$ такое, что $\left|\bar{f}_{\varepsilon}(x)-k_{\varepsilon}\right| \rightarrow 0$ равномерно в $\Omega$. Следовательно, $f-$ постоянная функция. 
Функции, лежашие в $W^{s, p}(\Omega)$, принадлежат пространству $\operatorname{VMO}(\Omega)$ при условии $s p \geqslant N$ (см. [10; $\S$ I.2]). Поэтому мы не можем непосредственно применить это рассуждение к нашей ситуации, которая соответствует, грубо говоря, случаю $s p \geqslant 1$. Однако мы можем воспользоваться методом сведения $к$ одномерному случаю, как уже делалось в [5].

Предположим, для простоты, что $\Omega$ - квадрат в $\mathbb{R}^{2}$. Пусть $f \in W^{s, p}(\Omega)$. Тогда ограничения $f\left(x_{1}, \cdot\right)$ и $f\left(\cdot, x_{2}\right)$ принадлежат пространству $W^{s, p}(I)$ при почти всех $x_{1}$ и почти всех $x_{2}$ (где $I$ - интервал) (см., например, [11; §2]).

Это наблюдение очень полезно в сочетании со следуюшим утверждением из теории меры.

Лемма (см., например, [11; лемма 2]). Пусть $f: \Omega \rightarrow \mathbb{R}$ измерима. Предположим, что при почти всех $x_{1}$ функция $f\left(x_{1}, \cdot\right)$ и при почти всех $x_{2}$ функция $f\left(\cdot, x_{2}\right)$ являются постоянными. Тогда $f$ - постоянна.

Приведенные вьше рассуждения влекут альтернативное доказательство следствия 1 при $p>1$. В самом деле, при $p>1$ условие (2) говорит, что $f \in W^{s, p}$, где $s=1 / p$. Ограничения $f$ на почти каждую прямую также принадлежат пространству $W^{s, p}$ c $s=1 / p$. Следовательно, эти ограничения принадлежат VMO.

Поэтому для отображения $f: \Omega \rightarrow \mathbb{Z}$ можно заключить, что ограничения $f$ на почти каждую прямую являются постоянными функциями. Приведенная выше лемма позволяет заключить, что $f$ - постоянная функция.

С помошью приведенного рассуждения можно получить следуюшую теорему.

Теорема 6. Предположим, ито $\Omega \subset \mathbb{R}^{N}$ - связна, и пусть $f: \Omega \rightarrow \mathbb{Z}-$ измеримая функиия такая, что $f=f_{0}+f_{1}+f_{2}+\cdots+f_{k}$, где $f_{0} \in W^{1,1}(\Omega ; \mathbb{R})$ u $f_{i} \in W^{s_{i}, p_{i}}(\Omega ; \mathbb{R})$ c $s_{i} p_{i} \geqslant 1$ при $i=1,2, \ldots, k$. Тогда $f-$ постоянная функиия.

ОТКРЫТАЯ ЗАДАЧА 3 . Имеется ли простое естественное условие на $f$, которое могло бы заменить предположение декомпозиции $f=f_{0}+f_{1}+f_{2}+\cdots+f_{k}$ ? Существует ли изящньй способ объединить теорему 6 с результатами $\S 1$.

Еще одним интересным направлением исследований является

ОТКРЫТАЯ ЗАДАЧА 4. Найти оценки для

$$
\left\|f-\int f\right\|
$$

в терминах величин, используемых в этой работе, которые в различных ситуациях влекли бы, что $f$ - постоянная функция. Некоторые результаты в этом направлении читатель может найти в [7] (см. также работу Мазьи и Шапошниковой [17]).

\section{СПИСОК ЛИТЕРАТУРЫ}

[1] R. A. Adams. Sobolev Spaces. New York: Academic Press, 1975.

[2] L. Ambrosio, P. Tilli. Selected Topics on "Analysis in Metric Spaces". Lecture Notes. Pisa: Scuola Normale Superiore Pisa, 2000.

[3] F. Bethuel, F. Demengel. Extensions for Sobolev mappings between manifolds // Calc. Var. Partial Differential Equations. 1995. V. 3. № 4. P. 475-491. 
[4] J. Bourgain, H. Brézis, P. Mironescu. Lifting in Sobolev spaces // J. Anal. Math. 2000. V. 80. P. 37-86.

[5] J. Bourgain, H. Brézis, P. Mironescu. On the structure of the Sobolev space $H^{1 / 2}$ with values into the circle // C. R. Acad. Sci. Paris Sér. I Math. 2000. V. 331. № 2. P. 119-124.

[6] J. Bourgain, H. Brézis, P. Mironescu. Another look at Sobolev spaces // Optimal Control and Partial Differential Equations. In honour of Professor A. Bensoussan's 60th Birthday / ed. J. L. Menaldi et al. Amsterdam: IOS Press, 2001. P. 439-455.

[7] J. Bourgain, H. Brézis, P. Mironescu. Limiting embedding theorems for $W^{s, p}$ when $s \uparrow 1$ and applications // J. Anal. Math. (to appear).

[8] H. Brézis. Analyse fonctionnelle. Théorie et applications. Paris: Masson, 1983; Paris: Dunod, 1999.

[9] H. Brézis, J.-M. Coron. Large solutions for harmonic maps in two dimensions // Comm. Math. Phys. 1983. V. 92. P. 203-215.

[10] H. Brézis, L. Nirenberg. Degree theory of BMO. I: Compact manifolds without boundaries // Selecta Math. 1995. V. 1. № 2. P. 197-263.

[11] H. Brézis, Y. Li, P. Mironescu, L. Nirenberg. Degree and Sobolev spaces // Topol. Methods Nonlinear Anal. 1999. V. 13. № 2. P. 181-190.

[12] J. Davila. On an open question about functions of bounded variation // Calc. Var. Partial Differential Equations (to appear).

[13] Д. Гилбарг, Н. С. Трудингер. Эллиптические дифференциальные уравнения с частными производными второго порядка. М.: Наука, 1989.

[14] P. Hajlasz, P. Koskela. Sobolev met Poincaré. Providence, RI: Amer. Math. Soc., 2000. (Mem. Amer. Math. Soc. № 688.)

[15] R. Hardt, D. Kinderlehrer, F.H. Lin. The variety of configurations of static liquid crystals // Variational Methods / ed. H. Berestycki et al. Boston: Birkhäuser, 1990. P. 115-131. (Progr. Nonlinear Differential Equations Appl. V. 4.)

[16] N. Korevaar, R. Schoen. Sobolev spaces and harmonic maps for metric space targets // Comm. Anal. Geom. 1993. V. 1. № 4. P. 561-659.

[17] V. Maz'ya, T. Shaposhnikova. On the Bourgain, Brézis and Mironescu theorem concerning limiting embeddings of fractional Sobolev spaces // J. Funct. Anal. (to appear).

Université Pierre et Marie Curie, Paris

E-mail: brezis@ann.jussieu.fr, brezis@ccr.jussieu.fr

Поступила в редакцию 05.04 .2002 